Meta

Journal des traducteurs

Translators' Journal

\title{
Perspectives d'avenir de la traduction automatique
}

\section{Karl-Heinz Brinkmann}

Volume 24, numéro 3, septembre 1979

URI : https://id.erudit.org/iderudit/002146ar

DOI : https://doi.org/10.7202/002146ar

Aller au sommaire du numéro

Éditeur(s)

Les Presses de l'Université de Montréal

ISSN

0026-0452 (imprimé)

1492-1421 (numérique)

Découvrir la revue

Citer cet article

Brinkmann, K.-H. (1979). Perspectives d'avenir de la traduction automatique. Meta, 24(3), 315-325. https://doi.org/10.7202/002146ar

\section{Résumé de l'article}

$\mathrm{Vu}$ le besoin pressant d'intensifier le transfert des connaissances et ce, au-delà des barrières linguistiques, il n'est pas étonnant que la traduction automatique fasse à notre époque l'objet d'un intérêt grandissant. L'auteur du présent article cherche à mettre en lumière les problèmes et les objectifs de la traduction, à juger d'après ces critères l'état actuel de la traduction automatique et à tirer des conclusions sur les tendances probables et souhaitables dans ce domaine. 


\section{Perspectives d'avenir de la traduction automatique*}

\author{
Résumé \\ Traduction \\ automatique, \\ aperçu ; \\ traduction
}

\begin{abstract}
$\mathrm{Vu}$ le besoin pressant d'intensifier le transfert des connaissances et ce, au-delà des barrières linguistiques, il n'est pas étonnant que la traduction automatique fasse à notre époque l'objet d'un intérêt grandissant. L'auteur du présent article cherche à mettre en lumière les problèmes et les objectifs de la traduction, à juger d'après ces critères l'état actuel de la traduction automatique et à tirer des conclusions sur les tendances probables et souhaitables dans ce domaine.
\end{abstract}

\section{Perspectives d'avenir de la traduction automatique}

Après la parution du rapport de l'ALPAC ${ }^{1}$, la recherche en traduction automatique (T.A.) arriva en 1966 à un point mort. Jusqu'alors, c'est surtout aux Etats-Unis que d'importantes sommes d'argent lui avaient été consacrées. Ả lui seul, le Gouvernement américain avait dépensé jusqu'en 1965 près de 20 millions de dollars (voir annexe 16 au numéro 1), sans que des résultats notables soient obtenus pour autant. Cinq ans plus tard, en 1971, a paru, à l'Université du Texas, l'étude intitulée Feasibility Study on Fully-Automatic High Quality Translation ${ }^{2}$ qui avait été commandée par l'U.S. Air Force. Les auteurs étaient unanimes pour constater : "The remaining problem is language»; toutefois, ils ne pouvaient se mettre d'accord sur la question de savoir si on pouvait obtenir une traduction de qualité en faisant appel à l'ordinateur. Manifes-

* Tiré à part de $<$ Nachrichten für Dokumentation $\$, 29 e$ année $(1978$, No 3, pages 104108)

ISSN 0027-7436 - Editions Dokumentation K.G. Saur, Munich - New York - London - Paris (autrefois Verlag Dokumentation Saur KG, Munich).

Publié avec l'autorisation du Bureau des traductions, Secrétariat d'Etat, Hull, qui a assuré la traduction du présent texte.

1. Language and Machines. Report by the Automatic Language Processing Advisory Committee (ALPAC) (Langue et machines. Rapport du comité consultatif du traitement automatique de la langue), National Academy of Sciences - National Research Council, Publication 1416, Washington (D.C.), 1966.

2. W.P. Lehmann et R. Stachowitz, Feasibility Study on Fully Automatic High Quality Translation. Final Technical Report (Etude de faisabilité sur la traduction de qualité complètement automatique. Rapport technique final), University of Texas, Linguistics Research Center, Austin, Texas 1971. 
tement, les spécialistes avaient du mal à définir le concept de high quality en matière de traduction. Ils ne partageaient pas non plus la même opinion sur la notion acceptable (machine) translation. Pourtant, ils étaient dans leur majorité d'accord pour dire que le refus des demi-mesures, qui avait caractérisé les premières recherches en traduction automatique, avait fait plus de mal que de bien.

Depuis environ trois ans, les recherches en traduction automatique reprennent de plus belle. Différentes universités se penchent sur les problèmes de la traduction automatique, fait qui passe presque inaperçu. Il s'agit, dans la plupart des cas, d'une recherche assez élémentaire concernant les objectifs mêmes de la traduction automatique : dans d'autres cas, on se limite à explorer certains aspects de la question. La mise en vente de systèmes à usage commercial ou qui visent un tel usage n'a repris, en tout cas, que tout récemment. Comme on sait, diverses institutions publiques aux États-Unis et ailleurs ont utilisé dans l'intervalle des systèmes de traduction automatique à certaines fins pratiques. Mais ce n'est que depuis deux ans environ que des institutions nationales et supranationales européennes commencent à mettre à l'essai des systèmes de traduction automatique.

\section{Pessimisme et euphorie}

Les points de vue sur la traduction automatique vont du pessimisme à l'euphorie. Le Pr. Krückeberg, président de la Gesellschaft für Mathematik und Datenverarbeitung (Société de mathématiques et de traitement des données), située à Bonn et où on a procédé à l'essai de tels systèmes, avait bien raison de faire la mise au point suivante : «Nous n'envisageons aucunement - dans cet essai - de mettre en question l'utilité de la traduction automatique et nous nous défendons de nous enthousiasmer outre mesure pour des succès partiels et, partant, d'imprimer de fausses directions à la traduction automatique. »

Dans tout cela, il est quand même frappant qu'on n'ait à peu près rien dit au sujet du genre de traduction qu'on a à l'esprit pour utiliser les procédés automatiques. Plus spécialement aux États-Unis, la traduction automatique servait avant tout à rendre des documents en langue étrangère accessibles, c'est-à-dire à les traduire dans la langue maternelle pour transmettre grosso modo leur contenu et non pas pour fournir des informations précises. Une exception : les Américains se sont efforcés, à la fin de la guerre du Vietnam, au cours de la " vietnamisation », de traduire dans la langue du pays les manuels d'emploi des armes. L'entreprise a réussi, mais seulement au prix d'un important travail de post-édition, qui a entraîné des dépenses considérables ${ }^{3}$. A condition d'envisager la traduction automatique comme un outil pratique d'information et de ne pas se soucier outre mesure des considérations stylistiques, il est permis de croire que la T.A., doublée d'un apport humain pour le travail de pré- ou de post-édition, est à la fois faisable et rentable. La plupart de ceux qui s'occupent aujourd'hui de traduction automatique confondent ce genre de traduction très spéciale avec la traduction tout court. Cela tient peut-être au fait que les traducteurs - ici 3. LOGOS Development Corporation, Corporate Profile (Profil dune société), Middletown
(N.Y.), 1977. 
comme ailleurs - ne parviennent pas à se faire entendre et que trop souvent, c'est à des informaticiens, des documentalistes ou d'autres encore, sans aucune formation ni expérience professionnelle en matière de traduction, que l'on laisse le soin de discuter la mise en œuvre des procédés de traduction automatique ou de régler des problèmes étroitement liés à la traduction.

\section{Problèmes d'une toute autre ampleur}

On n'a absolument pas tenu compte du fait que les problèmes de traduction ont une portée bien différente chez nous, en Europe, en particulier dans les pays exportateurs non anglophones. On en entend très peu parler dans les débats sur les procédés de traduction automatique. Ce n'est guère surprenant puisque les traducteurs, le plus souvent, n'y participent pas. C'est pourquoi nous avons l'intention ici de jeter un peu de lumière sur ce problème particulier.

Il serait superflu de s'étendre à nouveau sur le thème de l'explosion de l'information que l'on associe en général, à propos de ses répercussions sur le problème de la traduction, à l'accès à la documentation étrangère. Le grand public ignore cependant qu'un nombre croissant de documents publiés au pays doivent, eux aussi, être traduits. Il faut prendre en considération le fait que, par suite du progrès technique, la documentation qui accompagne le produit, la somme des renseignements écrits donc, représente une part de plus en plus grande du produit d'ensemble et que le produit lui-même, privé de cette volumineuse documentation, serait de nos jours inutilisable, voire inutile. Dans bien des cas, la documentation représente déjà plus de cinquante pour cent de la valeur marchande totale du produit, documentation comprise. Quand on pense que l'exportation constitue la moitié (et plus) du chiffre d'affaires de grandes branches de notre industrie, on peut se faire une idée assez juste de la quantité de documentation qui doit être préparée et aussi, le plus souvent, traduite. Cette tendance s'accentue étant donné qu'en général les connaissances (le know-how) doivent, de plus en plus, être exportées. Cela est devenu indispensable si nous voulons d'une part, soutenir la concurrence sur le marché mondial et survivre économiquement et, d'autre part, venir en aide au Tiers Monde dans sa lutte contre la famine, la misère et la maladie en accélérant son développement technique et culturel.

\section{La documentation chez Siemens : un million de pages}

Quelques données numériques suffisent à mettre en évidence l'ampleur prise par les problèmes de traduction. Prenons le cas de la fourniture d'installations pour une usine où qu'elle soit, disons dans un pays en voie de développement : la livraison «clefs en mains \& et la formation du personnel exigent une documentation dont le volume atteint quelques centaines de milliers de pages (dactylographiées A 4). La documentation relative au fonctionnement d'un ordinateur s'élève à quelques dizaines de milliers de pages et, en général, elle doit être mise à jour au moins deux fois par an. Une centrale moderne, nucléaire ou conventionnelle, nécessite également entre 70000 et 100000 pages de documentation qui, dans une proportion non négligeable, ne peut être utilisée qu'une seule fois, 
précisément pour une seule centrale. Il n'en va pas autrement pour les stations au sol qui maintiennent la communication avec des satellites, etc. La maison Siemens, par exemple, produit actuellement, d'après des calculs approximatifs, un million de pages de documentation par an pour accompagner ses produits, dont une grande partie, nous l'avons déjà mentionné, doit être traduite, dans la plupart des cas en plusieurs langues.

C'est là que se situe la différence avec le type de traductions effectuées jusqu'ici par les systèmes de traduction automatique auxquels on attribue, souvent plus ou moins tacitement, le pouvoir d'effectuer n'importe quelle sorte de traduction (à l'exception de la traduction littéraire bien sûr !). Or, pour le genre de traductions dont il a été question au paragraphe précédent, qui sont au moins aussi importantes que les traductions de documentation scientifique étrangère, la qualité joue un rôle primordial. Comme nous l'avons mentionné en début d'article, les responsables de la traduction automatique aux États-Unis n'ont pu s'entendre sur la définition du concept high quality translation. Dans le cas des traductions dont nous parlons ici, il est relativement facile d'apporter une défnition du degré de qualité que l'on recherche : la traduction doit se lire comme un texte rédigé dans la langue maternelle (ne pas poser de problèmes de compréhension) et transmettre, sans les déformer, toutes informations contenues dans le texte original.

\section{Des normes de qualité élevées}

Des normes de qualité très élevées s'appliquent non seulement à la traduction de la documentation accompagnant un produit destiné à l'exportation ou de connaissance transmises aux pays du Tiers Monde, mais aussi à tout texte qui, en raison de l'importance ou de la portée du contenu qu'on cherche à communiquer, ne saurait être traduit de manière approximative. Ce n'est pas par un hasard si justement les grands services de traduction, qui doivent satisfaire à ces exigences, n'ont pas attendu la mise au point de systèmes opérationnels de traduction et ont créé leurs propres instruments de travail en mettant au point et en installant des procédés automatiques d'aide à la traduction basés sur les banques de données terminologiques.

Dans le cas de la traduction automatique de littérature spécialisée, effectuée à des fins d'information générale, il est compréhensible que peu d'importance soit accordée à la qualité, autrement dit que le concept de «qualité » soit défini autrement, et ceci pour les raisons suivantes : on présume que seulement un peu plus de trois pour cent du flot des publications qui tombent sous la rubrique « littérature spécialisée » sont importants, qu'environ seize pour cent présentent un certain intérêt et que le reste est sans intérêt ${ }^{4}$. Si ces chiffres sont exacts, ce n'est que dans vingt pour cent des cas, au maximum, que le lecteur d'une traduction approximative produite par ordinateur désirera en obtenir une version plus précise. Par ailleurs, il faut tenir compte du fait qu'en Europe, ceux qui s'intéressent à la littérature spécialisée sont en général capables de lire dans la langue d'origine cinquante pour cent des textes qu'ils jugent intéressants.

4. Zeitschrift für Bibliothekswesen und Bibliographie (Revue de bibliothéconomie et de bibliographie) 22 (1975), Cahier 5 , p. 390 . 


\section{Le plus souvent, la traduction approximative d'un texte spécialisé est suffisante}

Si cela aussi est exact, une traduction approximative obtenue par des procédés automatiques, convient, dans quatre-vingt-dix pour cent des cas pour la littérature spécialisée. Il est à peu près certain que les besoins de pré- et de post-édition peuvent être maintenus à un niveau acceptable, ce qui rend très avantageux les coûts indiqués par les fournisseurs des systèmes de traduction automatique en ce qui concerne le travail de rédaction et d'édition effectué par l'homme. Quant à la proportion de littérature spécialisée lue par les intéressés dans la version originale chez nous, il est peut-être intéressant de voir quelle en est l'influence sur la composition des travaux qui sont confiés aux services de traduction des grandes entreprises. Au bureau de traduction de Siemens AG, par exemple, la part des traductions effectuées vers l'allemand s'élève à seulement quinze pour cent environ du volume total. Les traductions de littérature spécialisée ne représentent qu'une part infime de ce pourcentage, et ce malgré les efforts importants de Siemens AG dans le domaine de la recherche et du développement ${ }^{5}$.

En revanche, le haut niveau de qualité auquel nous avons fait allusion plus haut vaut pour les quatre-vingt-cinq pour cent qui restent, c'est-à-dire pour les traductions de l'allemand vers des langues étrangères. Pour que les traductions faites par ordinateur répondent à cette exigence, il faut engager des dépenses de post-édition très élevées. La révision de traductions de ce genre confiée à des ressources humaines représente en général cinquante pour cent environ du coût total de traduction. Les fournisseurs de systèmes de traduction automatique citent des chiffres tout à fait différents. Selon eux, les dépenses occasionnées par le travail de post-édition s'élèvent à $\mathrm{DM} 0,12$ la ligne dans le cas de traductions faites par l'homme et à DM 0,15 lorsqu'il s'agit de traductions automatiques. Ces chiffres manquent totalement de réalisme si l'on tient compte du degré de difficulté et du volume des textes dont il est question et, par ailleurs, du médiocre niveau de qualité des traductions automatiques, même de celles effectuées à des fins d'information générale.

\section{La réalité est bien différente}

La réalité semble quelque peu différente. Bar Hillel estimait, à l'époque du rapport de l'ALPAC, que l' ' exactitude » de la traduction automatique se situait entre 35 et $40 \%$ et qu'en l'espace de cinq ans (jusqu'à 1971 donc), elle atteindrait 50 à $55 \%{ }^{6}$. Cela signifie ni plus ni moins que 45 à $50 \%$ d'un texte traduit par ordinateur doit être corrigé du point de vue de la terminologie, de la grammaire, de la syntaxe et de la sémantique et ce, sans parler de la limpidité du texte ou des effets de style qu'il contient. En outre, on ne sait pas

5. R. Brée, Die Spezialbibliotheken und das Bundesförderungsprogram Information und Dokumentation (Les bibliothèques spécialisées et le programme fédéral pour la promotion de l'information et de la documentation) Arbeitsgemeinschaft der Spezialbibliotheken (Groupe d'étude des bibliothèques spécialisées), (ASpB), Berlin, 1976.

6. W. Wilss, Ubersetzungswissenschaft - Probleme und Methoden (La Science de la traduction - Problèmes et méthodes), Stuttgart, 1977. 
très bien si les pourcentages se rapportent au nombre de phrases qui doivent être corrigées quant au contenu, ou au nombre de mots qui doivent être changés ou transposés afin d'obtenir des phrases "correctes». Quoi qu'il en soit, Zarechnak (voir numéro 2) parle de $80 \%$ comme d'un objectif réaliste. Les optimistes eux, pensaient (et pensent toujours !) qu'on peut arriver à $95 \%$. Ce qu'ils ne mentionnent pas, ce sont les dépenses qui permettraient d'atteindre ce chiffre.

Il est bien connu qu'à partir d'un certain point, les coûts investis dans l'amélioration de la solution d'un problème complexe augmentent selon une courbe exponentielle, jusqu'à devenir exorbitants (voir le programme de division syllabique des mots où ce point se situe aux environs de 95 à $97 \%$, suivant la langue) ; il arrive aussi qu'en apportant trop d' « améliorations » on rende le résultat à nouveau médiocre. Cela s'applique notamment à la transcription automatique de mots anglais en symboles phonétiques : le degré d'exactitude qui est susceptible d'être atteint s'élève à $80 \%$ environ ' . Cet exemple démontre justement qu'il faut envisager la phrase dans le contexte, sans quoi aucune amélioration n'est possible. Or, tous les systèmes opérationnels de T.A. se bornent jusqu'ici à traduire phrase par phrase.

Pour les exemples qui viennent d'être cités (division syllabique des mots, transcription phonétique), il est très facile de calculer le taux d'exactitude, puisqu'on peut établir très clairement les fautes à partir d'une valeur de référence. Comme nous l'avons déjà mentionné, il n'en va pas de même pour les 《 fautes» de traduction automatique. En dépit du caractère discutable de la définition, on entend par « faute » chaque mot qui doit être changé, inséré ou retranché afin d'obtenir des phrases «exactes». Une phrase est dite exacte lorsqu'elle transmet correctement dans la langue d'arrivée l'information que contient la langue de départ, compte tenu bien entendu du domaine qui est traité let du contexte. Si on calcule les taux d'erreur sur la base de pages dactylographiées A 4 (qui contiennent à peu près 35 lignes chacune), ce qui correspond à environ 350 mots en anglais, ces taux représentent de 157 mots à 175 mots dans le cas de Bar Hillel, 70 mots dans le cas de Zarechnak et 17 mots pour œ qui est du pourcentage avancé par les optimistes.

\section{Il faut corriger chaque phrase}

Dans le cadre des essais en T.A. effectués par la Société de mathématiques et de traitement des données, les traductions de textes soumis par la société Siemens AG contenaient environ 90 fautes par page. Une étude menée aux Etats-Unis a démontré entre autres qu'un texte de quelque 100000 mots comprenait environ 7000 phrases. Selon le procédé de calcul exposé plus haut, cela correspond, à raison de 14 mots par phrase, à 25 phrases par page. Cest donc dire que, pour ce qui concerne les textes de Siemens AG soumis aux tests, il

7. E. Wolf, Vom Buchstaben zum Laut. Maschinelle Erzeugung und Erprobung von Umsetzautomaten am Beispiel Schriftenglisch - Phonologisches Englisch. (De la lettre au son. Fabrication mécanique et essai de transcripteurs automatiques : anglais écrit - anglais phonologique), Braunschweig, 1977. 
faudrait sans aucun doute corriger chaque phrase d'une façon ou d'une autre, ne serait-ce que pour atteindre un niveau de qualité tant soit peu acceptable.

Des dépenses engagées au titre de la correction et de la post-édition qui constitueraient $50 \%$ des coûts de traduction (traducteurs humains), sont considérées comme acceptables, avons-nous dit auparavant. Or, les bureaux de traduction qui ne sont pas liés à l'industrie ni à des institutions publiques ou autres ne sont satisfaits de ces $50 \%$ de l'honoraire que si la révision exige tout au plus «cinq corrections à la main par page ».

La post-édition n'est rentable que si la traduction répond au départ à certaines exigences minimales de qualité. Autrement, le réviseur aura tendance à reprendre la traduction dans son entier. Le service de traduction de Siemens a participé - il en a été question ailleurs - à l'essai d'un système de traduction automatique organisé par la Société de mathématiques et de traitement des données pour le ministère de la Recherche et de la Technologie : des textes russes portant sur l'informatique ont été traduits en anglais. Les résultats n'ont guère été satisfaisants, puisque le niveau moyen de qualité d'un traducteur débutant n'a même pas été atteint.

Un des experts britanniques indépendants dont les services ont été retenus par le bureau de traduction de Siemens a exprimé l'avis suivant : ... the process has not yet been developed to an extent which would make it useable. The material is, to a large extent, incomprehensible. The problems are widely spread and it would not help much to call upon translators to correct the worst passages. One would have hoped that the machine translation might have been of sufficient quality to assist scanning for important information. Even this modest standard is not achieved... » Un autre expert, américain cette fois, confiait : "The problems are several. The most disturbing patterns which emerge are that the Russian dictionary is beyond criticism in terms of the gross and serious errors which even a short passage like the one we have just analyzed reveals. The incorrect translations are not a matter of coincidence alone; phraseology is lacking and incorrectly translated.»

\section{C'est à peine si on demande l'avis des traducteurs}

Il ne faut pas passer sous silence le fait qu'on a décidé de mettre à l'essai certains systèmes de traduction automatique sans consulter les traducteurs ou en ne faisant appel à eux qu'à l'occasion. En République fédérale d'Allemagne, du moins, la décision de mettre ces systèmes à l'essai a été prise sans qu'aucun des grands bureaux de traduction de l'industrie n'ait voix au chapitre. La décision sur le choix du système à tester aurait sans doute été prise selon des critères recevables, et non uniquement d'après les arguments intéressés avancés par les fournisseurs, si l'on avait consulté auparavant les experts en la matière.

Quoi qu'il en soit, le système fonctionne peut-être mieux avec d'autres paires de langues ou lorsque les textes présentent moins de difficultés. Pourtant, les essais en question ont démontré que, pour amener le produit de la traduction automatique à un degré de qualité acceptable, le prix de la post-édition est prohibitif. 
Jusqu'ici, peu de changements se sont opérés, semble-t-il, depuis le rapport de l'ALPAC. Comme on sait, des systèmes informatisés d'aide aux traducteurs avaient été mis au point avant la parution du rapport, tendance qui s'est accentuée par la suite. Il s'agit de systèmes qui, grosso modo, à l'aide de données terminologiques mises en mémoire, fonctionnent pour ainsi dire au même titre qu'un «dictionnaire » multilingue; les traducteurs ont accès par les moyens les plus variés à cet ordinateur qui peut constituer une aide précieuse. Mentionnons entre autres systèmes l'EURODICAUTOM ${ }^{8}$ de la Commission des communautés européennes, le LEXIS $^{9}$ du Bureau des langues de la République fédérale d'Allemagne, le TERMIUM ${ }^{10}$ du Gouvernement canadien et le TEAM ${ }^{11}$ de la société Siemens AG. D'autres systèmes analogues d'aide automatique à la traduction (il n'est pas question ici de banques de données terminologiques utilisées à d'autres fins, par exemple à des fins de normalisation) sont en voie d'élaboration. Ces systèmes se limitent toutefois à offrir une aide terminologique ou autre au traducteur; ils ne fournissent pas de traduction au sens propre du mot.

\section{Place au scepticisme}

Parfois on entend dire que le développement de «systèmes de traduction de qualité entièrement automatiques » connaît un regain de vie. Il faut accueillir ce genre de propos avec réserve. Souvent les déclarations de ceux qui se font les champions de la traduction automatique témoignent de leur incompétence en matière de traduction proprement dite : «... notre système fournit des traductions qui peuvent être utilisées directement et qui rendent les mots contenus dans le texte beaucoup plus fidèlement que les traductions humaines. $\gg \mathrm{Ce}$ à quoi le traducteur ne peut que répondre : «bon, eh bien!»

De telles déclarations sont propres à engendrer l'idée, chez les interlocuteurs les moins renseignés, qu'on disposera bientôt d'une boîte noire où il suffira d'introduire le texte de départ d'un côté pour que la version fin prête dans la langue (et la qualité) voulue émerge de l'autre. Les défenseurs de la traduction automatique ne sauraient affirmer avec sérieux que de bons résultats sont possibles sans que des ressources humaines ne fassent un certain travail de retouche. De ce point de vue, il n'y a pas de traduction «complètement automatique »,

8. P. François, Terminologische Datenbank EURODICAUTOM der Kommission der Europäischen Gemeinschaften (La Banque des données terminologiques EURODICAUTOM de la Commission des communautés européennes), document no 2381/76 (exposé présenté à l'occasion du Colloque international de terminologie), Paris-La Défense, 1976.

9. K.E. Berner, \&as Lexikographie-Informations-System (LEXIS) der Sprachendienstes der Bundeswehr $\gg$ (Le Système de lexicographie et d'information (LEXIS) du service linguistique des Forces armées de la RFA), Der Sprachmittler (1976), no 3, Bonn.

10. R. Dubuc, " $A$ description of the Termium System of the Bank of Terminology $\$$ (Description du système Termium de la Banque de Terminologie), META Journal des traducteurs - Translators' Journal, Vol. 17, n⿳0 4, Montréal, 1972.

11. J. Schulz, Eine Terminologiedatenbank für Ubersetzer - Ab́ragemöglichkeiten im System TEAM» (Une banque de données terminologiques pour les traducteurs. Le système TEAM : possibilités de questions-réponses), Tiré de : 3. Europä ischer Kongress über Dokumentationssysteme und -netze - Uberwindung der Sprachbarrieren, Luxemburg (Troisième congrès européen sur les systèmes et les réseaux documentaires - Franchir les barrières linguistiques, Luxembourg), Editions Dokumentation, Munich, 1977 . 
mais seulement une traduction effectuée par l'homme avec l'aide d'un ordinateur, le degré d'aide apporté variant d'un système à l'autre. D'ailleurs, certains représentants de systèmes de traduction automatique l'admettent volontiers.

Les idées qui viennent d'être exposées ne doivent pas inciter les traducteurs à se bercer d'illusions, à s'imaginer que le sujet «traduction automatique » est dérisoire et que rien ne changera dans leur profession même s'ils emploient maintenant des systèmes d'aide à la traduction tels que les systèmes de traitement de textes et les banques de données terminologiques. Certains traducteurs se délectent encore à raconter de vieux calembours sur les aberrations des machines à traduire. Assez souvent ils citent des exemples qui sont certes bien trouvés, mais qui appartiennent au domaine de la fiction ou encore des phrases hors contexte qu'ils feraient mieux de ne pas mentionner, puisqu'ils ne pourraient pas non plus les traduire sans connaitre ce contexte (the pen is in the box / the box is in the pen).

\section{On redouble d'efforts en linguistique}

Il a été question ci-dessus des coûts de rédaction qui demeurent trop élevés. Le temps d'arrêt qui a suivi la parution du rapport de l'ALPAC a eu toutefois d'heureuses répercussions sur le domaine de la linguistique : on a redoublé d'efforts et on a acquis de nouvelles connaissances relatives à la nature et au fonctionnement de la langue comme instrument de communication. On a découvert entre autres que la langue constitue un système ouvert (open-ended) qui se soustrait largement à la mathématisation. C'est pourquoi on digère mal les propos des fournisseurs de systèmes de traduction automatique à ce sujet : « Etant donné que la langue possède un nombre limité d'unités lexicales (le vocabulaire) et un nombre limité d'algorithmes combinatoires (la syntaxe), l'usage de l'ordinateur en traduction est non seulement possible mais plein de promesses ${ }^{12} \gg$. Inutile de commenter cette affirmation. Une simple constatation : la langue est un ensemble vivant et le langage technique en particulier, dans plusieurs domaines, évolue à un rythme vertigineux non seulement en ce qui concerne le vocabulaire mais aussi les autres aspects (par exemple, la phraséologie, la syntaxe, la sémantique).

Le rapport de l'ALPAC a donné le coup d'envoi à de nouvelles recherches en linguistique et les résultats dans ce domaine sont pris de plus en plus en considération dans l'élaboration de nouveaux systèmes de T.A. Ainsi, il n'est pas du tout exclu que les traductions automatiques des années quatre-vingts soient acceptables tant du point de vue des dépenses de rédaction et de révision confiées à des ressources humaines que du point de vue de la qualité du produit, laquelle laisse à désirer actuellement. Les méthodes de travail du réviseur risquent de subir des transformations : le recours à des systèmes interactifs, c'est-à-dire des systèmes qui permettent de corriger la première version en dialoguant avec l'ordinateur (sur un écran, par exemple), facilitera les tâches de manière significative et accélérera le travail.

12. P.P. Toma, \&oftwarepaket mit Russischkenntnissen \$ (Ensemble de programmes avec des connaissances de russe), GMD-Spiegel (1975), no 3, Bonn. 


\section{L'homme, dernière instance de révision}

Il est inconcevable de vouloir se passer de l'homme comme dernière instance de révision dans le cas de traductions où la qualité est exigée, d'une part, pour les raisons déjà citées et, d'autre part, parce que les textes d'origine varient trop du point de vue qualitatif. On pourrait envisager éventuellement de rédiger les textes à traduire selon des règles extrêmement strictes pour que les textes de départ transmis à la machine présentent le moins d'ambiguïté possible. Ce procédé est déjà utilisé avec succès, notamment pour la traduction de résumés (abstracts) de publications concernant l'industrie textile ${ }^{13}$. Cependant, il est fort douteux qu'on puisse répandre l'usage de règles aussi strictes et les appliquer à des textes spécialisés appelés à être traduits. De sérieux efforts ont été entrepris aux États-Unis pour amener les auteurs de textes techniques et scientifiques à travailler en ce sens; il a bientôt fallu y renoncer car c'était peine perdue ${ }^{14}$.

Supposons qu'à bref délai les systèmes de traduction automatique fournissent les ébauches de traduction - travail qui revenait au traducteur - et que ce dernier doive se faire réviseur. Des questions de formation et d'aptitudes se posent, bien entendu. C'est là un problème auquel les écoles de traduction et les gens du métier devraient s'employer très prochainement.

\section{Les traducteurs compétents sont en nombre insuffisant}

On ne saurait affirmer que, par suite de cette nouvelle répartition des tâches, le nombre des postes diminuera. La réalité est bien différente : le nombre de traducteurs compétents se trouve constamment en deçà de la demande et les besoins, qui croissent continuellement, viendront aggraver cette situation. Evidemment, l'accent est mis sur l'adjectif «compétent », par lequel on entend non seulement les capacités linguistiques mais aussi des connaissances approfondies du domaine dont relève le texte à traduire. Il ne faut pas non plus perdre de vue le fait qu'un nombre très élevé de diplômés d'écoles de traduction n'embrasseront jamais la carrière de traducteur parce qu'ils ne répondent pas aux conditions exigées ou encore parce qu'ils ont fait leurs études dans un autre but que celui de devenir traducteurs. Les grands services de traduction se rendent compte, chaque fois qu'ils annoncent un poste vacant, que le nombre de postulants compétents est tout à fait insuffisant. Il faut donc rehausser le niveau de compétence des traducteurs, en particulier de la génération montante, afin de satisfaire à la demande actuelle et, en outre, de répondre aux exigences des années quatre-vingts.

Qu'on l'appelle traduction automatique ou aide à la traduction, la traduction assistée par ordinateur va vraisemblablement s'imposer. Dans quelle mesure l'ordinateur sera-t-il capable d'accélérer la traduction de qualité d'importantes quantités de textes dépendra toutefois de la disponibilité de réviseurs et de 13. J.M. Ducrot, The TITUS II System (Le Système TITUS II), Institut Textile de France,

14. S.C. Dodd, «Model English 》 (L'anglais modèle), tiré de : N.W. Locke, A.D. Booth : Machine Translation of Languages (Traduction automatique des langues), New York, 1955. 
traducteurs-rédacteurs. Les 300000 mots à l'heure indiqués comme rendement des systèmes de traduction automatique (chiffre intéressant, mais uniquement en ce qui concerne la dépense de temps de l'ordinateur) représentent quelque 850 pages $\mathrm{A} 4$ à réviser. Si les réviseurs (conformément à leurs rétributions actuelles) rédigeaient, par heure, une à trois pages d'une qualité analogue à celle offerte aujourd'hui par les ordinateurs, la proportion entre le rendement de la traduction automatique et celui de la post-édition serait, pour ce qui est du temps, de 1:850 ou, dans le meilleur des cas, de 1:280. De nos jours, le volume des textes à traduire suffit amplement à alimenter les ordinateurs de manière continue. Cependant, il est douteux que le nombre de réviseurs compétents puisse satisfaire au besoin qui se dessine. Il est presque absurde donc de vouloir augmenter le rendement des systèmes de traduction automatique. Par contre, il serait logique d'en améliorer le niveau qualitatif. Pour ce faire, il faudra s'armer de patience et surmonter des nombreuses difficultés.

KARL-HeINZ BRINKMANN 